\title{
Desde el ISET al InDRE. III. Instituto de Salubridad y Enfermedades Tropicales: crisis y renovación, 1965-1989
}

Jorge Alejandro Ramírez-Hernández, ${ }^{1}$ María del Carmen Guzmán-Bracho, ${ }^{2 *}$

Martha Eugenia Rodríguez-Pérez, ${ }^{3}$ Carlos Viesca-Treviño ${ }^{3}$ y José Alberto Díaz-Quiñonez

'Universidad Nacional Autónoma de México, Facultad de Medicina, División de Estudios de Posgrado; 'Instituto de Diagnóstico y Referencia Epidemiológicos "Manuel Martínez Báez"; ${ }^{3}$ Universidad Nacional Autónoma de México, Facultad de Medicina, Departamento de Historia y Filoșofía de la Medicina. Ciudad de México, México

\section{Resumen}

El artículo analiza la situación y los cambios efectuados en el Instituto de Salubridad y Enfermedades Tropicales entre 1965 y 1989 para transformarse en el Instituto Nacional de Diagnóstico y Referencia Epidemiológicos. Se identificantres grandes etapas en el periodo: crisis, transición y renovación. Se señalan los elementos que llevaron a la crisis, las decisiones que se tomaron para salir de ella y armonizar el trabajo de los laboratorios con criterios epidemiológicos $¥$ de salud pública. Se citan las distinciones obtenidas por investigadores del Instituto a pesar de la crisis y se describe cómo la institución logró seguir con proyectos a pesar de la cambiante situación mundial. La transición incluyó la llegada dęuna nueva generación de profesionales con modernas herramientas informáticas y conceptuales y el desafío de participar en encuestas nacionales con criterios rigurosos. Todo ello movió a la institución a definir su perfil hacia el diagnósticơ y la referencia.

PALABRAS CLAVE: Epidemiología. Instituto de Salubridad y Enfermedades Tropicales. Instituto Nacional de Diagnóstico y Referencia Epidemiológicos. Historia de la medicina. Historia de la salud pública.

\section{Abstract}

This paper analyzes the situation and the changes made in the Institute of Sanitary and Tropical Diseases between 1965 and 1989 to become the National Institute of Epidemiological Diagnosis and Reference. Three major stages are identified during this period: crisis, transition and renewal. The factors that led to the crisis, the decisions made to overcome itand to harmonize the work of laboratories with epidemiological and public health criteria are discussed. The recognition obtained by researchers of the Institute despite the crisis is described, as well as the way the institution managed to continuegwith projects despite the changing global situation. The transition included the arrival of a new generation of professionalswith modern computer-based and conceptual tools and the remarkably well met challenge of participating in national surveys with rigorous criteria. All this moved the institution to define its profile towards diagnosis and reference.

KEYWORDS: Epidemiology. Institute of Sanitary and Tropical Diseases. National Institute of Epidemiological Diagnosis-and Reference. History of medicine. History of public health.

Correspondencia:

*María del Carmen Guzmán-Bracho

E-mail: cguzmanbracho@ hotmail.com
Fecha de recepción: 04-06-2019

Fecha de aceptación: 30-07-2019

DOI: 10.24875/GMM.19005318
Gac Med Mex. 2019;155:641-646

Disponible en Pǘmed

www.gacetamedicademexico.com 


\section{Introducción}

Este artículo da continuidad a la revisión de la vida del Instituto de Salubridad y Enfermedades Tropicales (ISET) entre 1965 y 1989,1,2 cuando se manifestó la crisis del modelo institucional y se tomaron decisiones para redireccionarlo.

En esos años, el perfil epidemiológico mostró cambios importantes para la salud pública del país, desafiantes para las políticas públicas. En las tablas de mortalidad se modificó el predominio de enfermedades infecciosas al de enfermedades no transmisibles y accidentes (Tablas 1 y 2), sin embargo, las diarreas y las neumonías siguieron como causas principales de morbilidad. La esperanza de vida de 1965 a 1989 a nivel nacional ascendió de 58.9 a 70.46 años, mayor el incremento en mujeres que en hombres (13 años más para mujeres y 10 más en hombres) (Figura 1). ${ }^{3-5}$

\section{La época de crisis del ISET}

La crisis de la institución la ubicamos entre 1965 y 1977, años de poco dinamismo e innovación. Un ejemplo de la crisis lo ilustra el testimonio de Clara Gorodezky. En 1965 se entrevistó con el director para incorporarse al ISET. En respuesta, él la recomendó al laboratorio de Investigaciones Inmunológicas, explicándole que en el ISET "solo había actividades rutinarias y escasa investigación” (Clara Gorodezky, comunicación personal).

En este lapso, además, se retiraron o murieron investigadores fundadores del Instituto. Luis Mazzotti, Eustaquio Roch, Miguel E. Bustamante y Carlos Campillo Sainz dejaron el ISET para atender otras labores profesionales; Manuel Martínez Báez se retiró de la vida institucional en $1969,{ }^{6,7}$ si bien conservó una oficina ahí (Juan Manuel Campos, comunicación personal). Fallecieron Eliseo Ramírez Ulloa (1940), José Zozaya (1957), Mario Salazar Mallén (1976), Gerardo Varela (1977) y Antonio González Ochoa (1984). Esta generación no transmitió su legado a otra más joven para renovar al Instituto. Para 1960, el salario de los investigadores, decoroso en su origen, ${ }^{1}$ era ya insuficiente. Los jóvenes con vocación y deseo para investigar se dirigieron a otras instituciones. ${ }^{8}$

Se registraron otros cambios importantes, como la transformación de la Revista del Instituto de Salubridad y Enfermedades Tropicales (Revista ISET). Para 1966, al cumplir 27 años, se le dio un nuevo nombre: Revista de Investigación en Salud Pública, que se mantuvo
Tabla 1. Quince principales causas de muerte de 1970 a $1975^{3}$

\begin{tabular}{|c|c|c|c|}
\hline Núm. & 1970 & 1975 & 5 \\
\hline 1 & Influenza y neumonías & Influenza y neumonías & ¿ \\
\hline 2 & $\begin{array}{l}\text { Enteritis y otras } \\
\text { enfermedades diarreicas }\end{array}$ & $\begin{array}{l}\text { Enteritis y otras enfermed } \\
\text { diarreicas }\end{array}$ & dades \\
\hline 3 & $\begin{array}{l}\text { Accidentes o muertes } \\
\text { violentas }\end{array}$ & Accidentes o muertes vio & plentas \\
\hline 4 & Enfermedades del corazón & Enfermedades del coraz & $o n \frac{0}{\bar{U}}$ \\
\hline 5 & $\begin{array}{l}\text { Causas de morbilidad y } \\
\text { mortalidad perinatales }\end{array}$ & $\begin{array}{l}\text { Causas de morbilidad y } \\
\text { mortalidad perinatales }\end{array}$ & $\frac{.0}{\frac{0}{0}}$ \\
\hline 6 & Tumores malignos & Tumores malignos & $\stackrel{0}{\tilde{U}}$ \\
\hline 7 & Bronquitis & $\begin{array}{l}\text { Enfermedades } \\
\text { cerebrovasculares }\end{array}$ & $\frac{0}{0}$ \\
\hline 8 & $\begin{array}{l}\text { Enfermedades } \\
\text { cerebrovasculares }\end{array}$ & Cirrosis hepática & $\stackrel{2}{\stackrel{2}{c}}$ \\
\hline 9 & Sarampión & Diabetes mellitus & $\overrightarrow{0}$ \\
\hline 10 & Cirrosis hepática & Bronquitis & 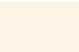 \\
\hline 11 & Tuberculosis & Tuberculosis & $\frac{}{2}$ \\
\hline 12 & Avitaminosis & Avitaminosis & 은 \\
\hline 13 & Diabetes mellitus & Infecciones respiratorias & agüdas \\
\hline 14 & Anemias & Anemias & $\cong$ \\
\hline 15 & Anomalías congénitas & Anomalías congénitas & $\frac{2}{2}$ \\
\hline
\end{tabular}

hasta 1977, cuando fue incorporada a la Revista de Salud Pública de México. ${ }^{9}$ Este hecho permitió aceiccar a otras dependencias de la Secretaría de Salubridad y Asistencia para publicar. La denominación sería cercana al nombre que adoptaría esta (Secretaría de Salud, 1982) y al del futuro Instituto (Instituto Nacional de Salud Pública, 1987). El concepto de salud pública permitió al ISET vincularse a un movimiento mundial más amplio que el de la medicina tropical y fue cơherente con los posgrados en salud pública de cincō de sus nueve médicos fundadores. ${ }^{1,10}$

La crisis institucional también se enfrentó de otras formas: a principios de la década de 1970, al interior del ISET se creó el Centro Nacional de Diagnóstico y Referencia bajo la dirección de David Bessüu Madjar, para coordinar laboratorios que funcionaban aislados entre sí, homogenizar sus procedimieñtos técnicos y de información en acuerdo con el ărea epidemiológica. ${ }^{11,12}$ De esta forma, se permitió la participación más adecuada del ISET ante brotes eppidémicos, como el de fiebre tifoidea por salmonella resistente a cloranfenicol en $1972 .^{13-15}$

En la segunda mitad de 1970, el perfil dễlos profesionales de laboratorio fue cambiando con la 


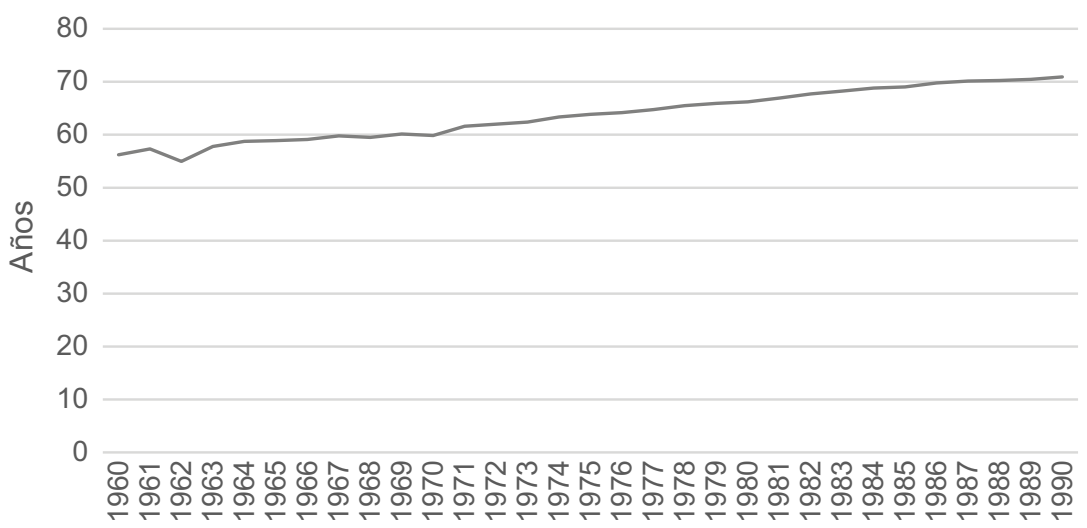

Figura 1. Esperanza de vida al nacimiento en México 1960-1990. Datos disponibles en Consejo Nacional de Población. Indicadores demográficos de México de 1950 a 2050.

Tabla 2. Principales causas de muerte de 1979 a 1989 en México

\section{Núm. Causa}

Causas de muerte comunes en el periodo

1 Infarto agudo del miocardio

2 Bronconeumonía sin mención de desnutrición

3 Otras armas de fuego y las no especificadas

$4 \quad$ Cirrosis hepática sin mención del alcoholismo

5 Colitis, enteritis y gastroenteritis infecciosa

6 Neumonía, organismo causal no especificado

7 Paro cardiaco

8 Colitis, enteritis y gastroenteritis de presunto origen infeccioso

9 Insuficiencia cardiaca sin especificación

10 Senilidad sin mención de psicosis

11 Diabetes mellitus sin mención de complicación

12 Cirrosis hepática alcohólica

13 Otras causas accidentales, accidentes no especificados

14 Tuberculosis pulmonar, sin especificación

\section{Causas de muerte incorporadas al final del periodo}

1 Enfermedad cerebrovascular aguda pero mal definida

2 Otras formas de desnutrición proteinocalórica sin especificación

3 Síndrome de dificultad respiratoria

$4 \quad$ Peatón

Fuente: Cubos dinámicos Instituto Nacional de Estadística y Geografía (INEGI)-Secretaría de Salud.

incorporación de jóvenes químicos, Para 1977 ya era similar la cantidad de médicos y químicos en el ISET (58\% versus $42 \%$, respectivamente), ${ }^{16}$ perfil que incluía el de dos directivos: David Bessudo Madjar y
Adolfo Pérez Miravete (Luis Ángel Sapian, comunicačión personal) (Tabla 3$)^{6}{ }^{6}$

En conclusión, durante la crisis se produjo un intento programado y también azaroso para dinamizar los laboratorios seleccionados. Por ejemplo, se inició la producción de antisueros para tipificar enterobacteriās y bajo la conducción de Enriqueta Pizarro Suárezoccomenzó el Laboratorio de Virus Respiratorios, en apoyo a la vigilancia epidemiológica de influenza. ${ }^{17,18}$

Sin embargo, lo anterior no fue suficiente para reorientar las labores del ISET, que seguía en una sifuación muy difícil. Se conoce el dramático testimonio narrado por Miguel E. Bustamante a Antonio Goñzález Ochoa, cuando defendió al ISET ante la ameñaza de cancelar sus actividades y preguntó al Secreaario de Salubridad y Asistencia: "¿quiere usted pasara la historia como el secretario que cerró el ISET?...". evitó el fin de actividades del Instituto (Alejandro Escobar, comunicación personal).

En este difícil trance se confirieron varias distinciones a miembros del ISET, inercia de sus mejores momentos. En 1972, Antonio González Ochoa recibió el Bremio Nacional de Ciencias y Artes y en 1977, la medalla de oro "Eduardo Liceaga".19 En 1975, Gerardo Varela fue galardonado en la primera edición del Premio $\mathrm{Na}$ cional de Química y Ciencias Farmacéuticas. En ëste periodo, algunos recipiendarios del Premio "Gerărdo Varela" habían sido investigadores del ISET: Jorge Olarte, 1976; Luis Vargas, 1978 y Jorge Fernándež de Castro, 1988, ${ }^{20}$ impulsor inicial de la renovación del ISET como director general de epidemiología.

\section{Movimiento internacional de la salud públíca}

En la segunda mitad de 1970 surgieron nuevas propuestas de la Organización Mundial de la Salud: Salud 
Tabla 3. Directores del Instituto de Salubridad y Enfermedades Tropicales, 1965-19896

\begin{tabular}{|l|c|}
\hline Director & Periodo \\
\hline Gerardo Ignacio Varela Mariscal & $1959-1977$ \\
\hline Antonio González Ochoa* & $1975-1977$ \\
\hline Diego Fernández de Castro & $1977-1979$ \\
\hline Pablo Mendoza Hernández & $1980-1981$ \\
\hline Eduardo Zorrilla Hernández & $1981-1984$ \\
\hline David Bessudo Madjar* & $1984-1985$ \\
\hline Adolfo Pérez Miravete & $1985-1990$ \\
\hline *Directores interinos & \\
\hline
\end{tabular}

para Todos en el Año 2000 (1977) y la Conferencia Internacional sobre Atención Primaria de Salud en Alma-Ata (1978). Se añadió a este panorama dar a conocer los últimos casos de viruela por infección natural en el mundo.

Con un incremento de recursos internacionales debido al aumento de los precios del petróleo, en 1974 se organizó un programa de interés para el ISET: el Tropical Diseases Research. El Laboratorio de Investigaciones Inmunológicas recibió apoyo para desarrollar proyectos en oncocercosis, línea de trabajo institucional iniciada desde la década de 194016,21 (Alejandro Escobar, comunicación personal).

En 1981, el panorama global optimista se ensombreció con la abrupta caída de los precios internacionales del petróleo. Para 1982, México realizó su ajuste estructural, disminuyeron los presupuestos y los enfoques del sector salud se volvieron selectivos. ${ }^{22}$ EI ISET continuó su impulso renovador y se centró en programas de vacunación; contribuyó con el estudio de la respuesta inmune protectora y de los eventos temporalmente asociados con la vacunación. ${ }^{23}$

\section{Transición del ISET hacia el INDRE}

\section{El Centro Nacional de Diagnóstico y Referencia del ISET}

Al inicio del periodo que nos ocupa, el diagnóstico de las enfermedades virales recibió un enorme impulso para establecer la etiología y los mecanismos de transmisión de las enfermedades y se actualizaron las acciones preventivas y curativas. ${ }^{24,25}$

En este sentido, el Centro Nacional de Diagnóstico y Referencia se integró por laboratorios para enfermedades virales y bacterianas de importancia en salud pública, además de la Unidad de Epidemiología
(David Bessudo Madjar, comunicación personal). Las muestras procedían de las unidades médicas (centros de salud y hospitales) que participaban en los programas especiales de vigilancia epidemiológica. A dỉagnóstico se agregó el desarrollo de estudios epidem̄iológicos especiales. ${ }^{26}$ En la reestructuración de la Secretaría de Salubridad y Asistencia en 1977, este centro se consideró parte fundamental del ISET $\mathrm{T}_{\sigma-2}$ incluso se propuso cambiar nombre al instituto; éxito, a Centro para la Investigación de Enfermedades Transmisibles. ${ }^{27}$

El 5 de diciembre de 1977 se firmó el acta de entrega del ISET y del Laboratorio de Investigaciones Inmưnológicas a la Dirección General de Epidemiologĩa e Investigación en Salud Pública; ${ }^{28}$ en 1978, el laboratörio pasó a depender del ISET (si bien, se integró a las instalaciones del Instituto de Diagnóstico y Refereñcia Epidemiológicos [INDRE] hasta 1992). ${ }^{6}$ Sus actividades se enriquecieron con la incorporación de laboratorios con experiencia como el Centro de Diagnóstico y ferencia de Enfermedades Venéreas en abril de 1978.29

Otra medida integradora del ISET con la Dirección General de Epidemiología fue ubicar a la Subdirección de Investigación Epidemiológica en el Instituto y a un grupo de epidemiólogos recién graduados de la Escuela de Salud Pública de México que abriêron nuevas líneas de investigación. Al mismo tiempo se inició el Curso de Especialización en Laboratorió de Infectología en Salud Pública, con visión amplià de las redes de laboratorio en la salud y el manejo epidemiológico del padecimiento e innovación tecnológica para el diagnóstico (Jorge Ramírez Hernándejz y Carmen Guzmán Bracho, comunicación personal).

En 1984, en el ISET se comenzó a evaluar la dînámica de transmisión de las helmintiasis transmitidas por suelo, ${ }^{30}$ base del Programa Nacional de Despărasitación en la década de 1990. En 1985, el ISĒ se convirtió en el primer laboratorio del país con pruebas para confirmar los casos de infección por el viruš de la inmunodeficiencia humana (Roberto Vázquez Campuzano, comunicación personal).

El Instituto recibió recursos y equipo; después del sismo de septiembre de 1985 se aceleró la remodelación del inmueble. Se creó el Banco de Sueros, que centralizó el manejo de cerca de 70000 muestras obtenidas durante la Encuesta Nacional Seroepidemiológica, para el estudio inicial de nueve enfermedades, dentro y fuera del Instituto, definidas en función dë su importancia en salud pública, así como la factibilidad para ser prevenidas o controladas: infecciones bactêrianas (tosferina, brucelosis), virales (sarampión, rubeola y 
poliomielitis), parasitarias (cisticercosis, chagas, toxoplasmosis) y metabólicas (colesterol total sérico). ${ }^{31}$ Esta encuesta fue de gran importancia para dar un nuevo impulso al ISET, con un equipo profesional joven, bien calificado, que dio certeza al diagnóstico en salud. Se impulsó el desarrollo tecnológico, por ejemplo, para tipificar Vibrio cholerae 01, Salmonella y Brucella. La serología tuvo un avance importante en la Encuesta Nacional Seroepidemiológica, al estandarizar metodologías propias, recibir capacitación especializada en centros de referencia internacional e incorporar profesionales con formación en áreas específicas.

\section{La evolución de un aspecto crucial: la vigilancia epidemiológica en el ISET}

Es importante hacer una descripción del elemento central en la nueva orientación del ISET para transformarse en Instituto de Diagnóstico y Referencia Epidemiológicos: la integración del laboratorio con la vigilancia epidemiológica.

Desde 1956, la Organización Mundial de la Salud promovió reuniones de expertos para definir la organización y funcionamiento de los laboratorios de salud pública en cada país. ${ }^{32}$ EI ISET cumplió a cabalidad los criterios emitidos en 1970 para que fuera designado como laboratorio central. Así, en 1974 se planteó la primera etapa de formación de una red de laboratorios con dependencia técnica del Centro Nacional de Diagnóstico y Referencia del ISET. ${ }^{12,33,34}$ Los primeros laboratorios, en Toluca y Guadalajara, iniciaron funciones en la primera mitad de la década de $1970 .{ }^{35,36}$

Como consecuencia del proyecto de descentralización de los servicios de salud, se promovió la creación de laboratorios estatales de salud pública, en detrimento del proyecto de laboratorio regional. A partir de la segunda mitad de la década de 1970 hasta 1989 entraron en funciones 11 laboratorios más: Yucatán, Guanajuato, Quintana Roo, Guerrero, Colima, Puebla, Tabasco, Chihuahua, Nuevo León, Zacatecas y Aguascalientes. De esta manera, el diseño piramidal de laboratorios se fue integrando por todo el país. Con ello, desde finales de 1980 se comenzó a desarrollar, desde la salud pública, la contraparte al Plan Nacional de Hospitales de 1940.

\section{Cambio del ISET al INDRE}

Con todo lo expuesto, fue evidente la reorganización del Instituto. El inmueble recibió una remodelación a fondo desde 1985 y las autoridades, dentro y fuera del ISET, discutieron la idea de convertirlo en el Instituto Nacional de Diagnóstico y Referencia Epidemiológicos (INDRE). Finalmente, después dê 50 años de vida, en 1989 se llevó a cabo el cambiē de denominación. EI ISET se transformó en INDRE, con un programa de trabajo de gran impacto en la vigitancia epidemiológica nacional y el gran reto de recuperar y fortalecer su credibilidad.

\section{Discusión}

El propósito de este artículo ha sido ofrecer tuna visión de la evolución institucional del ISET. Para èste periodo de estudio se contó con el testimonio personal de al menos un participante de cada área-del Instituto, que complementó la revisión documental en 13 archivos y hemerotecas históricos.

Estos años (1965 a 1989) presentaron características propias para su análisis. La Revista ISE L̆ se transformó en Revista de Investigación en Sălud Pública y para 1977 se incorporó a la Revistà de Salud Pública de México. El cambio de nombrę de la revista trascendió al ISET, ${ }^{10}$ afectaría al conjünto de la Secretaría de Salud y del sector salud en México. Los conceptos de salubridad y enfermedades tropicales fueron menos empleados y sustituidosapor los de salud o salud pública. Hemos descrito olas fuerzas que influyeron para llevar a crisis al ISET y las decisiones tomadas para resolverlo. Un punto de importancia fue renovarlo con equipos de profesionales jóvenes y redefinir su vocación hacia el diagnóstico y la referencia epidemiológicos, conceptos que ya se habían utilizado en el ISET desde la década de 1970, con la idea de establecer laboratớpios de salud pública, regionales o estatales. Esta acción dio equilibrio al binomio epidemiología-laboratơrio, para convertirse más tarde en la actividad centraह̄del Instituto.

En 1985 se decidió cerrar la clínica del ISET cuando ya se definían nuevas funciones del Instituto. Actualmente, el ausente componente clínico debe desarrollado en protocolos de colaboración con institutos nacionales de salud.

El impulso en la formación de recursos humaños jóvenes especializados en epidemiología y laboratorio de salud pública, así como el uso de nuevas teęnologías informáticas fueron cruciales para orientar los programas de prevención y control de enfermedades. Lo anterior permitió lograr confianza hacia la institución, donde fue fundamental la Encuesta Naciōnal Seroepidemiológica. 
En 1989, el Instituto de Salubridad y Enfermedades Tropicales se transformó en Instituto Nacional de Diagnóstico y Referencia Epidemiológicos, lo que significó pasar de ser un instituto acotado a un edificio a convertirse plenamente en la cabeza de la compleja red de laboratorios para el diagnóstico en enfermedades infecciosas sujetas a vigilancia epidemiológica en México.

\section{Agradecimientos}

A las autoridades y personal del Archivo Histórico de la Secretaría de Salud y a los entrevistados: David Bessudo Madjar(†), Juan Manuel Campos Cervantes, Alejandro Escobar Gutiérrez, Clara Gorodezky Lauferman, Roberto Vázquez Campuzano y Luis Ángel Sapian López.

\section{Bibliografía}

1. Ramírez-Hernández JA, Guzmán-Bracho C, Díaz-Quiñonez JA. 2019. Desde el ISET al InDRE. I. Instituto de Salubridad y Enfermedades Tropicales: génesis y primeros años, 1934-1940. Gac Med Mex. 2019;155(3):322-327. DOI: 10.24875/GMM.19005157.

2. Ramírez-Hernández JA, Guzmán-Bracho C, Viesca-Treviño C, Díaz-Quiñonez JA. Desde el ISET al InDRE. II. Instituto de Salubridad y Enfermedades Tropicales: Madurez y consolidación, 1940-1964. Gac Med Mex. 2019;155:391-398

3. Almada-Bay I. La mortalidad en México. México: Instituto Mexicano del Seguro Social; 1982.

4. Defunciones cifras oficiales definitivas. Cubos de defunciones 19792017. México: Instituto Nacional de Estadística y Geografía/Secretaría de Salud; 2019.

5. Consejo Nacional de Población. Indicadores demográficos de la República Mexicana, en el año. México: Consejo Nacional de Población; 2019

6. Valdespino-Gómez JL, García-García ML, Del Río-Zolezzi A, Escobar A Mora JL. El Instituto Nacional de Diagnóstico y Referencia Epidemiológicos "Dr. Manuel Martínez Báez" en 1994. En: Una institución académica y dieciséis investigadores distinguidos. Quincuagésimo quinto aniversario del INDRE. México: Secretaría de Salud; 1994

7. Mazzotti L. Nota necrológica. Salud Publica Mex. 1971;13:983-984

8. Archivo Histórico. Fondo Secretaría de Salubridad y Asistencia c44e1. México: Secretaría de Salud; 2019.

9. Marcial LD. Las publicaciones oficiales de la Secretaría de Salubridad y Asistencia. Salud Publica Mex. 1983;25:489-497.

10. Bustamante ME, Viesca-Treviño C, Villaseñor F, Vargas-Flores A, Castañón R, Martínez-Barbosa X. La salud pública en México 1959-1982. México: Secretaría de Salubridad y Asistencia; 1982.

11. Archivo Histórico de la Secretaría de Salud. Fondo Instituto de Salubri- dad y Enfermedades Tropicales c9e6. México: Secretaría de Salud;:

12. Archivo Histórico. Fondo Instituto de Salubridad y Enfermedades Jropicales c23e1. México: Secretaría de Salud; 2019.

13. Fernández-De Castro J, Fernández-De Castro, H. Endemias y epidemias de México en el siglo XX. México: Siglo XXI Editores; 2015.

14. Pérez-Miravete A. A program for epidemiologic investigation of infections caused by Shigella dysenteriae type 1 in Mexico. Bol Oficina Sanit Panam. 1973:6:44-50.

15. González-Cortés A, Bessudo D, Fragoso R, Hinojosa M, Becèrril P Sánchez Leyva R, et al. Water-borne transmission of chlorampheniêl-resistant Salmonella typhi in Mexico. Bull Pan Am Health Organ. 1974;302:605-607.

16. Archivo Histórico de la Secretaría de Salud. Fondo Secretaría deşalubridad y Asistencia c19e1. México: Secretaría de Salud; 2019.

17. Archivo Histórico de la Secretaría de Salud. Fondo Instituto de Sałubridad y Enfermedades Tropicales c1e13. México: Secretaría de Sălud; 2019

18. Archivo Histórico de la Secretaría de Salud. Fondo Instituto de Sălubridad y Enfermedades Tropicales c23e4. México: Secretaría de Salud; 2019.

19. Bonifaz-Trujillo A. Micología. En: Méndez-Oteo F. Historia gráfica de la medicina mexicana del siglo XX. México: Méndez Editores; 2019.0

20. Premios que otorga el Consejo de Salubridad General. México: Consejo de Salubridad General; 2019.

21. Archivo Histórico de la Secretaría de Salud. Fondo Secretaría de Salubridad y Asistencia c2e4. México: Secretaría de Salud; 2019.

22. Walsh JA, Warren KS. Selective primary health care. New Engl FMed. 1979:30:1967-1974

23. Archivo Histórico de la Secretaría de Salud. Fondo Instituto de Salubridad y Enfermedades Tropicales c22e2. México: Secretaría de Salud; 2019.

24. Archivo Histórico de la Secretaría de Salud. Fondo Secretaría de Salubridad y Asistencia c45e2. México: Secretaría de Salud; 2019.

25. Archivo Histórico de la Secretaría de Salud. Fondo Secretaría dëSalubridad y Asistencia c1e2. México: Secretaría de Salud; 2019.

26. Archivo Histórico de la Secretaría de Salud. Fondo ISET/Direeción c26e6. México: Secretaría de Salud; 2019.

27. Archivo Histórico de la Secretaría de Salud. Fondo Secretaría de Salubridad y Asistencia. Resumen del Programa de Trabajo de la Dirección de Investigación en Salud Pública. México: Secretaría de Salud; I9.

28. Archivo Histórico de la Secretaría de Salud. Anexo del oficio Subsecretaría de Salubridad 116/032. México: Secretaría de Salud; 1978.

29. Archivo Histórico de la Secretaría de Salud. Oficio Subsecretafa de Salubridad 021/0359. México: Secretaría de Salud; 1978.

30. Lara-Aguilera R, Vallejo-Aguilar O, Gómez-Rojo E, Guzmán-Bracho C. Albendazol en el tratamiento de infecciones simultáneas de Trichuris trichiura y otros helmintos intestinales en niños. Bol Med Hosp tnfant Mex. 1984;41:539-544.

31. Magos-López C, Sánchez-Villarreal F, Gutiérrez G, Tapia-Conyer R. Banco Nacional de Sueros. Salud Publica Mex. 1992;34:136-147을

32. The public health laboratories services: first report of the Expert Committee on Health Laboratory Methods. Suiza: World Health Organization; 1957.

33. Ávila-Cisneros I. La especialización de los laboratorios de saludiy su coordinación e integración. Salud Publica Mex. 1968;10:421-424.(1)

34. Borunda-Falcón $\mathrm{O}$. Nuevos laboratorios regionales de salud públiea; su importancia en los programas de vigilancia epidemiológica. Salud.Publica Mex. 1974;16:59-66.

35. Dirección de Laboratorios de Salud Pública-CESSLAB. México: Šecretaría de Salud; 2013.

36. Laboratorio Estatal de Salud Pública. México: Instituto de Salü del Estado de México; 2019. 